\title{
Mechanical multi-function buttons in intelligent building installations as a way to reduce electricity consumption
}

\author{
Mateusz Sałuch ${ }^{1}$, Robert Tomaszewski ${ }^{1}$, Bartosz Zegardło $^{1}$, Wojciech Andrzejuk ${ }^{1}$, \\ Zofia Lubańska ${ }^{1}$ \\ ${ }^{1}$ Faculty of Economic and Technical Sciences, Pope John Paul II State School of Higher Education \\ in Biała Podlaska, Poland
}

\begin{abstract}
This article aims to show the possible energy savings in intelligent building installations due to the use of programmable mechanical multifunctional buttons. Two monostable buttons were placed, the pressing of which triggered the entire sequence of programmed actions and procedures. The consumption of electricity from the period before and after the assembly of the buttons was compared. The measured effects of using the buttons have shown that the energy consumption has decreased by approximately $7.5 \mathrm{kWh}$ per month. The low installation costs and benefits resulting from the use of such a solution make it a cost-effective and a very convenient solution.
\end{abstract}

\section{Introducion}

The research was aimed at showing the possibility of saving electricity in intelligent building installations due to the use of programmable mechanical multifunctional buttons. The main assumption of the research was to check how such buttons placed in the crucial points of the building - at the exit door and in the bedroom next to the bed, affect the reduction of electricity consumption. The installation used during the tests was designed and made for a five-room apartment. (living room, bedroom, kitchen, bathroom and hall).

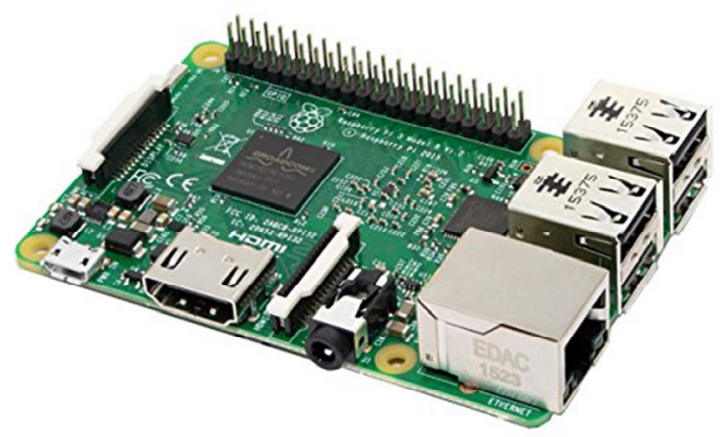

Fig. 1. Raspberry Pi 3B + module. 
The installation allowed one to control the lighting, electrical sockets and elements connected to them, electrically heated floors in the bathroom, heating, blinds and to monitor the conditions prevailing in the apartment (temperature, humidity, flooding, movement) [1]. The "heart" of the above-mentioned installation is the Raspberry Pi 3B + microcomputer (Figure 1) along with the Domoticz software [2]. In contrast, the executive devices were modules based on AtMEGA 2560 microcontrollers as well as semiconductor and mechanical relay modules controlled by them. All sensors have also been connected directly to the AtMEGA microcontroller.

Lighting control is possible both with the use of monostable buttons placed in the wall in the place of ordinary installation connectors and with the help of an application available on almost all hardware platforms or websites. The control of electrical sockets is carried out only through an application or a website. Readings from all sensors are currently sent to the Domoticz software [3], where it is possible to perform almost any interpretation, create statistics and take actions based on the values of the parameters read. The control of the heating mat on the bathroom floor is carried out using an SSR contactor designed to control devices up to $50 \mathrm{~A}$. The heating mat is switched on when the readings from the two temperature sensors installed in the bathroom are lower than the set value of the first temperature threshold in the Domoticz software. Switching on the heating mat is also limited by the operation time, because the mat is activated only in the morning (before the residents leave for work or school) and in the afternoon and evening hours. Outside these hours, the heating mat will turn on only when the bathroom temperature falls below the second temperature threshold - a value lower than the first threshold. This proceeding allows for a significant reduction of electricity consumption without causing loss of comfort in the bathroom and without manually switching on the heating mat prior to use.

The use of motion sensors allowed taking programmed actions after detecting motion in a given room. This action allows turning on the hall light for a pre-programmed time when movement is detected, for example when entering through the entrance door or when going to the bathroom or kitchen. After the programmed time expires, the light automatically switches off without having to press any buttons. In the case of other sensors, no action is taken after the motion is detected, only the date and time of the motion sensor operation is saved.

It should be remembered that the tests have been carried out with the use of an already existing smart home installation. The resulting differences in energy consumption will not be as drastic as in the case of exchanging the usual installation for the installation of a smart home. However, it can be noticed that the slight interference in the installation (installation of two buttons) allows you to reduce the consumption of electricity without having to give up the comfort of use. 
Table 1. Basic parameters of Raspberry Pi 3B + microcomputer and AtMEGA 2560 microcontroller [5].

\begin{tabular}{|l|l|}
\hline \multicolumn{2}{|c|}{ Rasperry Pi 3B+ } \\
\hline Parameter & Description \\
\hline Processor & $1.2 \mathrm{GHz}$ \\
\hline Number of cores & 4 Quad Core \\
\hline The numer of cores RAM & $1 \mathrm{~GB}$ \\
\hline Memory & MicroSD memory card \\
\hline GPIO & 40 \\
\hline USB & 4 \\
\hline HDMI & 1.4 Standard \\
\hline Communication & Ethernet, Wi-Fi, Bluetooth \\
\hline Protocols & UART, I2C, SPI \\
\hline System & System \\
\hline \multicolumn{2}{|c|}{ AtMEGA 2560 microcontroller } \\
\hline Operating voltage & $5 \mathrm{~V}$ \\
\hline Frequency & $16 \mathrm{MHz}$ \\
\hline SRAM memory & $8 \mathrm{kB}$ \\
\hline Flash memory & $256 \mathrm{kB}$ \\
\hline EEPROM memory & $4 \mathrm{kB}$ \\
\hline Number of inputs / outputs & 54 \\
\hline Number of PWM outputs & 15 \\
\hline Number of analog inputs & 16 \\
\hline External interrupt & Yes \\
\hline Protocols & UART, I2C, SPI \\
\hline
\end{tabular}

\section{Description of conducted research}

The research was carried out in two stages. The first stage consisted of measuring the consumption of electricity during normal use of the apartment with the installation of the intelligent building described in the introduction. The meter reading was performed at the same time every day. After one month, the last reading of the electricity consumption was made. The next step was the assembly of two mechanical multifunctional buttons - one at the exit door and one above the bed in the bedroom. Both functions have been assigned to both buttons and actions to be performed after pressing. The exit door button turns off all lights and all controlled electrical sockets, this causes the temperature in the bathroom to move to the second temperature threshold - lowering the temperature. In addition, when motion is detected after pressing the button, the user will be notified about it by e-mail. To turn off the entire sequence after entering the apartment, all you have to do is press the button again. The second button placed next to the bed causes the installation to go to the night mode, i.e. all lights and controlled electrical sockets are turned off and the bathroom cools down to a lower programmed temperature. In contrast to the first button, we can still control lighting, sockets, etc., after the motion is detected, the user will not be informed by e-mail. When the sensor is activated in the hall, the light will automatically turn on for the programmed time. As you can see, the programmes of both buttons are optimized on account of switching off devices that the user is not using. Such an operation allows limiting the consumption of electricity and other types of energy, e.g. heat energy. The programme of both buttons has been adapted to the preferences of the apartment residents in order to achieve the most reliable results. 
The next step was to train residents about the operation, functionality and the use of the buttons. Then, as in the first stage, the measurement of electricity consumption was made at the same time every day. The last measurement was made after 30 days from the beginning, i.e. exactly as many days as during the previous month. The tests were carried out in December and in January, so both temperature and other weather conditions were very similar. The results obtained are presented in Figure 2.

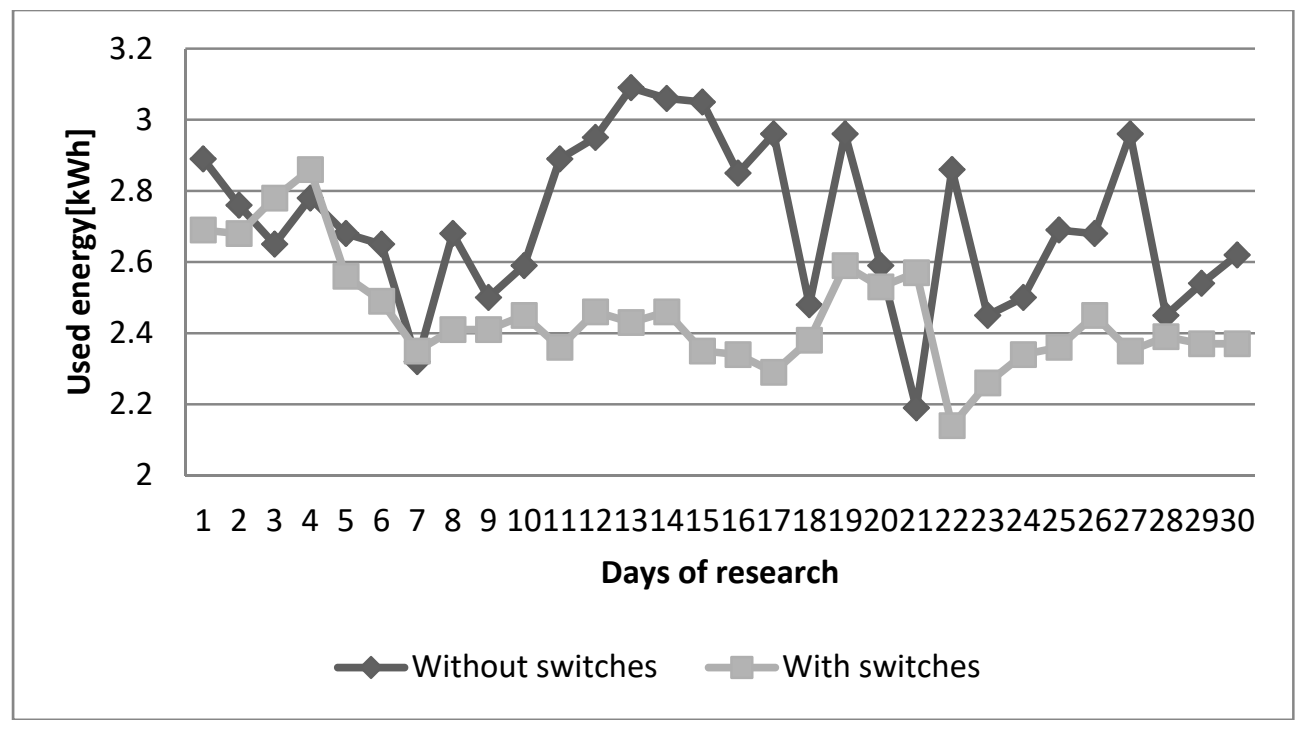

Fig. 2. Electricity consumption during subsequent days of the week.

Monthly electricity consumption of a smart home installation alone without the use of multi-function buttons was $81,322 \mathrm{kWh}$, which itself is quite a good result with an average electricity consumption of $110 \mathrm{kWh}$ per month. The additional installation of two multifunctional buttons enabled the reduction of energy consumption to the level of $73.47 \mathrm{kWh}$, i.e., $7.85 \mathrm{kWh}$ less was consumed in the month. So as it can be observed, the simple modernization of the installation allows one to save even more energy without having to give up any comfort of using the apartment [4]. It should be remembered, however, that the obtained results could have been influenced by other factors such as the current weather, outdoor temperature, amount of rainfall and many other factors, e.g. the need to consume more hot water or leave the light turned on all night.

Another aspect of the conducted research was the estimation of the costs of button assembly and their programming. The entire assembly operation lasted about an hour and required only the purchase of two monostable buttons and twenty meters of a two-core cable with a core cross-section of $0.5 \mathrm{~mm}^{2}$. The cost of the entire installation was estimated at 50 PLN. Assuming, therefore, an average saving of approximately $7.5 \mathrm{kWh}$ per month, with a power supply at the level of 0.55 PLN per $1 \mathrm{kWh}$, gives us savings of 4.13 PLN. The value of the pointer SPBT is - 12.1. As you can see, the purchase and assembly costs of such equipment will pay the consumer back after about twelve months. However, it should be remembered that the above-mentioned buttons do not only serve to save electricity, but also improve the comfort of living and improve the security of the apartment while the users are not present. 


\section{Conclusions}

The research was aimed at showing the possibility of saving electricity in intelligent building installations due to the use of programmable mechanical multifunctional buttons. Multi-stage studies show that in the month scale, $7.85 \mathrm{kWh}$ less electricity was consumed, which results in real savings of 4.13 PLN per month. The value of the pointer SPBT is only - 12.1. Improving the comfort of living and improving the security of the apartment, while all users are not in the apartment is just an additional aspect for the installation of buttons with the functionality described in the article. Even a small modification of the smart home installation parameters allows not only to improve the comfort of use and safety, but also to reduce electricity bills.

\section{References}

1. J. Kwaśniewski, Inteligentny dom i inne systemy sterowania w 100 przykładach, (Wydawnictwo BTC, 2015)

2. M. Riley, Smart home. Automation of flats using the Arduino platform, android system and ordinary computer, (Wydawnictwo HELION, 2014)

3. M. Schwartz, Home automation witch Arduino, (Wydawnictwo HELION 2014)

4. E. Niezabitowska, Budynek inteligentny. Tom I. Potrzeby użytkownika a standard budynku inteligentnego, (Wydawnictwo Politechniki Śląskiej, Gliwice, 2014)

5. Nota katalogowa mikrokomputera Raspberry Pi 3B+ 